\title{
Medienwechsel und seine Folgen für das Recht und die rechtswissenschaftliche Methode
}

\author{
Thomas Vesting
}

\section{Medienwechsel als umstürzende Fundierungsphase}

Die Erfindung und Ingebrauchnahme neuer Medien ging in der Vergangenheit stets mit einem Wandel der kulturellen Symbolsysteme der Gesellschaft einher. Von diesem kulturellen Wandel waren immer auch die Bestände des gemeinsamen Wissens betroffen. Das gilt nicht nur für theoretisches Wissen, für das, was gedacht und gesagt werden kann, sondern auch für praktisches Wissen, für das, was Menschen durch Kunstfertigkeit machen und hervorbringen können. Die griechische Philosophie, insbesondere die platonische Kunst der Unterscheidung, das dihairetische (dialektische) Verfahren der Begriffseinteilung, ${ }^{1}$ wäre ohne die griechische Alphabetschrift, die vermutlich von Töpfern als Inschrift erfunden wurde, ${ }^{2}$ nicht möglich gewesen. ${ }^{3}$ Und der so frühe Triumph über die Hungersnot in England am Ende des 16. Jahrhunderts hätte ohne die Verbreitung von preiswerten gedruckten Broschüren zur Effektivierung der Bodenbewirtschaftung, die die englischen Bauern mit Kenntnissen und Erfahrungen des agrarischen Wissens der Holländer vertraut machte, wohl nicht stattgefunden. ${ }^{4}$

1 Vgl. etwa Platon, Sophistes 218b-221c (am Beispiel der Angelfischerei); dazu Julius Stenzel, Studien zur Entwicklung der platonischen Dialektik von Sokrates zu Aristoteles (1931), 1974.

2 Vgl. die Diskussion bei Eric A. Havelock, The Muse learns to Write, New Haven (Conn.) 1986, 79ff., 85; und Kevin Robb, Literacy and Paideia in Ancient Greece, New York 1994, 21ff., 44ff. Die weit verbreitete Annahme, dass die Verschriftlichung der Homerischen Epen der Auslöser für die Erfindung/Übernahme der Alphabetschrift im frühantiken Griechenland war, dürfte kaum haltbar sein.

3 Kevin Robb, Literacy and Paideia in Ancient Greece, Oxford 1994, insb. 265ff.; Christian Stetter, System und Performanz. Symboltheoretische Grundlagen von Medientheorie und Sprachwissenschaft, 2005, $117 \mathrm{ff}$.

4 Joyce O. Appleby, The Relentless Revolution. A History of Capitalism, New York 2010, $73 \mathrm{ff} ., 76$. 
In beiden Fällen hatte der Medienwechsel weitreichende Konsequenzen für die Dynamik des Westens: Ohne die durch die Alphabetschrift möglich werdende platonische Kunst begrifflicher Unterscheidungen wäre wiederum das römische Recht mit seiner elaborierten Scheidekunst und seinen Klassifikationen wohl niemals zu dem Corpus juristischen Wissens geworden, zu dem es zunächst im republikanischen Rom wurde und seit der Spätantike dann für ganz Westeuropa geworden ist. ${ }^{5}$ Und ohne die Beseitigung der Hungersnot durch gedruckte Broschüren hätte es den vielleicht größten Umbruch in der jüngeren Menschheitsgeschichte wiederum nicht gegeben: Die britische industrielle Revolution. Es spricht zwar nicht sehr viel für die These, dass der industriellen Revolution eine agrikulturelle Revolution vorausgegangen sei. ${ }^{6}$ Die relativ frühe Beseitigung der Hungersnot und die Tatsache, dass um 1700 nur noch ein Drittel der Briten von der Agrikultur lebten, stellt aber doch eine prä-adaptive Vorentwicklung im Sinne der Theorie der kulturellen Evolution dar. Diese Vorentwicklung hat die industrielle Revolution insofern mitermöglicht, als auf ihrer Grundlage ein ganz unwahrscheinlicher Umbruch der gesellschaftlichen Wissensarchitektur möglich wurde: der Aufstieg einer „Kultur der Neugierde", 7 in der nützliches Wissen, technologische Innovationen, Offenheit, demographischer Wandel und ökonomisch effektive Institutionen sich gegen die alte christlich-aristotelische Wissensordnung durchsetzen konnten. ${ }^{8}$ Auf diesem Durchbruch basiert die bis heute anhaltende explosionsartige Wohlstandsvermehrung auf dem gesamten Globus.

Wenn der Medienwechsel in der Vergangenheit stets einen Wandel der kulturellen Symbolsysteme und der Wissensordnung zur Folge hatte, an den sich mehr oder weniger umstürzende Veränderungen in unterschiedlichen gesellschaftlichen Handlungsfeldern und Institutionen angeschlossen haben, dann liegt die Vermutung nahe, dass auch der Übergang zu einer informationstechnologischen Kultur, ${ }^{9}$ deren Zeugen wir seit den späten

5 Vgl. Aldo Schiavone, The Invention of Law in the West, Cambridge (Mass.) 2012, 154ff., 175ff., 184ff.; Thomas Vesting, Die Medien des Rechts, Bd. II: Schrift, 2011, 139ff.; Fabian Steinhauer, Vom Scheiden. Geschichte und Theorie einer juristischen Kulturtechnik, 2015, insb. 68ff.

6 Vgl. Joel Mokyr, The Enlightened Economy: An Economic History of Britain, 1700 - 1850, New Haven (Conn.) 2009, $171 \mathrm{ff}$.

7 Joanna Picciotto, Labors of Innocence in Early Modern England, Cambridge (Mass.) 2010, 255ff.; vgl. auch Joel Mokyr, A Culture of Growth. The Origins of the Modern Economy - the Graz Schumpeter Lectures, Princeton (N.J.) 2017.

8 Vgl. Mokyr, The Enlightened Economy, 196.

9 Weitere Hinweise dazu bei Thomas Vesting, Staatstheorie, 2018, 157ff. (unter dem Begriff der Netzwerkkultur). 
1960er Jahren sind, weitreichende Konsequenzen nicht nur für die Wirtschaft, sondern auch für das Recht haben wird. Aber wie kann man diese Zusammenhänge beschreiben? Wo und wie erfassen die digitalen Technologien das Recht und transformieren es? Und wie könnte eine der informationstechnologischen Kultur adäquate Rechtsform aussehen - adäquat auch in dem Sinn, dass sie als Rechtsform zu einer „entwicklungsadäquaten Gerechtigkeit" ${ }^{10}$ eben dieser Kultur beitragen könnte?

Diese Fragen stellen einen Aufsatz vor ein schwer lösbares Problem. Meines Erachtens kann die Frage, welche Folgen der jüngste Medienwechsel für das Recht haben wird, allein durch das Sammeln von empirischen Erfahrungen in einzelnen gesellschaftlichen Handlungsfeldern und rechtlichen Referenzgebieten, die bereits heute sichtbar von den digitalen Technologien betroffen sind, nicht erfasst werden. Auch die neuen technologischen Möglichkeiten, die beispielsweise mit der künstlichen Intelligenz verbunden sind, setzen schon eine Idee davon voraus, wie sich das Recht verändern könnte, bevor man das Neue, das bislang Unbekannte - die Veränderung - beschreiben kann. Man muss also zunächst eine Intuition dafür entwickeln, wonach man sucht, erst dann können in einem Handlungsfeld und Referenzgebiet des öffentlichen Rechts wie dem der Medienverfassung, der Gesundheitsversorgung, der Bankenregulierung oder der Schul- und Universitätsorganisation Material gefunden und Entwicklungen beobachtet werden, die eine zu formulierende Grundhypothese über die Folgen des Medienwechsels für das Recht stützen oder gegen sie sprechen.

Die Formulierung oder Suche nach einer solchen Idee ist der Kern theoretischer Arbeit. In der theoretischen Arbeit wird auch die Art des Zugriffs auf die durch die informationstechnologische Kultur ausgelösten Veränderungen festgelegt, das „Vorverständnis“, mit dem die neue Problemlage angegangen werden soll. Das setzt eine Reihe von Überlegungen und Schritten voraus, die hier aufgrund des gewählten Formats des wissenschaftlichen Aufsatzes übersprungen werden müssen. Mein Zugriff, soviel sei aber zu einer groben Orientierung vorausgeschickt, setzt nicht, jedenfalls nicht primär, bei den möglichen Folgen der informationstechnologischen Kultur für die moderne Gesellschaft an. Es geht mir beispielsweise nicht um die Beschreibung und Analyse einer neuartigen „environmentali-

10 Rudolf Wiethölter, Materialisierungen und Prozeduralisierungen im Recht, in: Zumbansen/Amstutz (Hrsg.), Recht in Recht-Fertigungen, 2014, 423. 
tären Situation" im Sinne Michel Foucaults, ${ }^{11}$ um eine Tiefentransformation, die die einst disziplinierenden und normalisierenden Macht/Wissenskomplexe der staatlichen Souveränität in neuartige selbstlernende Kontrollsysteme environmentaler Technologien verwandelt; es geht mir auch nicht um die Transformation von normativen zu smarten Ordnungen, in denen mittels künstlicher Intelligenz die Verhaltenskontrolle der Einzelnen in allen möglichen Alltagssituationen optimiert und der Gebrauch individueller Urteilskraft und Entscheidungsfreiheit möglicherweise eingeschränkt oder sogar suspendiert wird. ${ }^{12}$ Stattdessen setzen meine Überlegungen auf der Ebene des Machens ein. ${ }^{13}$ Das impliziert vor allem die praktische Seite der informationstechnologischen Kultur, die Erzeugung neuen Wissens und die materiell fundierten Prozesse in den Blick zu nehmen, die die neuen Informationstechnologien überhaupt erst ermöglichen. Die Rechtstheorie wird dann vom materiellen Kontext der informationstechnologischen Kultur abhängig, und verliert damit die Souveränität über ihre Fragestellung. Wie das moderne Subjekt die Grammatik der Sprache, in der es spricht, vorfindet und Subjektivität daher bereits bei Wittgenstein als grammatische (und nicht mehr als autonome) Subjektivität gedacht wurde, ${ }^{14}$ so ist das begriffliche Rechtsdenken notwendigerweise auf ein „Erfahrungsfeld“ angewiesen, das seinerseits auf die reale Welt eines „es gibt" verweist. ${ }^{15}$ Und diese Wirklichkeit wird gerade im Moment ihrer Überschreitung, etwa durch die Erfindung einer neuen Technologie

11 Vgl. Michel Foucault, Die Geburt der Biopolitik. Geschichte der Gouvernementalität II, 2006, 359ff., wo Foucault die Grenzen der auf innerliche Unterwerfung programmierten Disziplinarmacht reflektiert und einen neuen Macht-Wissenskomplex umreißt, den er „Environmentalität“ nennt; daran anknüpfend Erich Hörl, Die environmentalitäre Situation. Überlegungen zum Umweltlich-Werden von Denken, Macht und Kapital, Internationales Jahrbuch für Medienphilosophie 4 (2018), 221, $230 \mathrm{ff}$.

12 So Klaus Günther, Von normativen zu smarten Ordnungen, Manuskript 2020.

13 Vgl. Tim Ingold, Making. Anthropology, Archaeology, Art and Architecture, London 2013; und Victoria Kahn, The Trouble with Literature, New York 2020, 25, 38 (die, in historischer Perspektive, von "maker's-knowledge" spricht).

14 Dazu Sandra Markewitz, Das grammatische Subjekt. Konstitutionsformen von Subjektivität in der Moderne, in: dies. (Hrsg.), Grammatische Subjektivität, Bielefeld 2019, 23; vgl. auch Ino Augsberg, Verantwortung als Reflexion, Rechtswissenschaft 2019, 109, 119 (der dieses „Je-schon-ausgesetzt-Sein“ des Subjekts als Bruch oder Spaltung des Subjekts beschreibt, die als Voraussetzung aller Selbstkonstitution erfahren wird).

15 Gilles Deleuze/Félix Guattari, Was ist Philosophie?, 2018, 22. 
„nicht begrifflich artikuliert, sondern erprobt und erfahren“ ${ }^{16}$ Der nächste Abschnitt versucht, den mit diesen knappen Hinweisen verbundenen Grundgedanken meines (rechts-)theoretischen Zugriffs auf den aktuellen Medienwandel zu veranschaulichen.

\section{Softwaredesign: Sam Ginn als Beispiel}

Sam Ginn ist 22 Jahre alt und einer der Jungstars unter den Entwicklern künstlicher Intelligenz in Stanford, einer der Top-Universitäten der Welt in diesem Feld. Ginn arbeitet im Bereich der Computerlinguistik, an der Schnittstelle von Sprachwissenschaft und Informatik. Auf Sam Ginn bin ich durch einige Artikel in der Neuen Züricher Zeitung und durch HansUlrich Gumbrecht aufmerksam geworden. Gumbrecht ist ein deutscher Literaturwissenschaftler, der viele Jahrzehnte in Stanford gelehrt und eine Fülle von Erfahrungen mit hochtalentierten jungen Informatikern wie Sam Ginn gesammelt hat. Ginn ist mit Gumbrecht in Kontakt gekommen, weil er sich für deutsche Literatur interessiert und insbesondere ein Faible für Musil, Heidegger und Nietzsche hat. Ginns Ziel ist es, eine allgemeine künstliche Intelligenz zu entwickeln, die wie die menschliche universal einsetzbar ist. ${ }^{17}$ Es geht also um eine artifizielle, elektronische Subjektivität und Maschinenintelligenz, die es heute (noch) nicht gibt, die aber schon länger das Leitbild für die vor allem in Nordamerika angesiedelte Forschung zur künstlichen Intelligenz darstellt. ${ }^{18}$ Was zeichnet Softwaredesigner wie Sam Ginn aus? Was ist die Besonderheit der Software-Produktion, der heutigen ökonomischen Kernkompetenz des Silicon Valley? Über welche Fähigkeiten muss man verfügen, um darin besonders erfolgreich zu sein? Ich beschränke mich auf zwei Aspekte und folge dabei den Analysen, die Gumbrecht aus Gesprächen mit Sam Ginn und anderen gewonnen hat.

16 Karl-Heinz Ladeur, „Same sex marriage ... is still a novel concept” Familienrecht ohne Vater?, Manuskript 2020, 10; vgl. dazu auch allgemein Hans-Jörg Rheinberger, Experimentalität. Hans-Jörg Rheinberger im Gespräch über Labor, Atelier und Archiv, 2018.

17 Vgl. das Interview von René Scheu mit Sam Ginn in der NZZ vom 14.6.2019; und ders., „Wir erschaffen eine künstliche Superintelligenz, die selbst lernt“. René Scheu spricht mit Sam Ginn in Stanford, in: Gumbrecht/Scheu (Hrsg.), Weltgeist im Silicon Valley. Leben und Denken im Zukunftsmodus, Zürich 2018, 221.

18 Vgl. aus philosophischer Perspektive Catharine Malabou, Morphing Intelligence. From IQ Measurement to Artificial Brains, New York 2019, 86ff. 
Zum einen benötigen Softwaredesigner eine scharfe formale Intelligenz. Man muss ein brillanter Mathematiker und Informatiker sein, aber das propositionale Wissen allein genügt nicht. Am Anfang einer jeden Aufgabe, die Softwareentwickler mittels eines Algorithmus zu lösen versuchen, steht eine Überkomplexität von Verfahrensmöglichkeiten. Die Komplexität dieser Möglichkeiten ist so groß, dass sie in rein rationaler Weise nicht zu reduzieren oder gar produktiv zu lösen ist; die Anfangsbedingungen können niemals mathematisch vollständig abgeleitet werden. Auch im weiteren Verlauf des Schreibens eines Softwarecodes geht der jeweils durchzuführende mathematische Schritt der Möglichkeit seiner Registrierung durch das Bewusstsein voraus. Selbst im Rückblick lassen sich Lösungen und Entdeckungen nicht als vorausgeplante Leistungen des Programmierers beschreiben. ${ }^{19} \mathrm{Um}$ erfolgreich zu sein, muss es vielmehr zu einer Verknüpfung von Rationalitäten kommen, zu einer, wie Gumbrecht sagt, „Kopräsenz verschiedener Denkformen“. ${ }^{20}$ Das bedeutet, dass sich verschiedene Denkformen wechselseitig füreinander öffnen müssen. Softwaredesigner müssen zu einer Art des Denkens in der Lage sein, das, so erneut Gumbrecht, der Kontemplation nahekomme, einer fokussierten und zugleich entspannten Konzentration, die offen für das Unerwartete der eigenen Intuitionen und das unerwartete Andere sei. ${ }^{21}$

In einer kantischen Terminologie könnte man sagen, dass die reine Vernunft durch die Urteilskraft und ihr Herzstück, die Einbildungskraft, unterlegt werden muss. Während die mathematische Kompetenz eine Nähe zur reinen Vernunft zeigt, zeigt die entspannte Konzentration eine Nähe zur Imagination, zu intuitiven Vorgriffen auf die Zukunft durch Bilder, Metaphern und Visionen. ${ }^{22}$ Zur Arbeitsform der Softwaredesigner gehört neben den formal-logischen Kompetenzen die Fähigkeit, mit unbekannten Möglichkeiten zu spielen und sich auf ein unbekanntes Terrain zu begeben, auf dem man auf das unerwartet Andere trifft. Das entscheidende Moment der Arbeitsform der Softwaredesigner liegt also darin, dass es zwischen der Absicht, ein Programm zu schreiben, und dem fertigen Produkt einen nicht kalkulierbaren ereignishaften Schritt gibt, der zum Erfolg oder Misserfolg der Ausgangshypothese führt. Der französische Philosoph und Wissenschaftstheoretiker Michel Serres beschreibt dieses Moment des Nichtvorwegnehmbaren technischer Erfindungen so:

19 Vgl. Gumbrecht, in: ders./Scheu (Hrsg.), Weltgeist im Silicon Valley, 73.

20 Gumbrecht, ebd., 85.

21 Gumbrecht, ebd., 84.

22 Gumbrecht, ebd., 84. 
„Keine Wissenschaft ohne Technik, ohne Maschinen, vor allem nicht ohne ... Computer. Jeder glaubt und hat tausend Gründe, daß ihre Erfinder - von Leibniz und Pascal bis zu Turing und von Neumann - sie fertig im Kopf hatten, ehe sie darangingen, Prinzipien, Geräte und Programme zu entwickeln. Nein. Wer forscht, weiß nicht, sondern tastet sich vorwärts, bastelt, zögert, hält seine Entscheidungen in der Schwebe. Nein, er konstruiert den Rechner von übermorgen nicht; während wir, die wir ihn kennen und fortan benutzen, leicht dem Fehlschluss erliegen, er habe ihn vorausgesehen. ${ }^{23}$

Es ist daher eigentlich unzutreffend, Sam Ginn einen Softwaredesigner oder Programmierer zu nennen. Software ist zwar ein Resultat menschlichen Handelns, aber eben nicht das Resultat eines konstruktiven Entwurfs, eines Designs, einer Planung, eines Programms oder einer Gesetzmäßigkeit im strengen Sinn. In einer phänomenologischen Terminologie könnte man formulieren, dass das erfolgreiche Schreiben von Algorithmen das Machen neuer Erfahrungen voraussetzt. Neue Erfahrungen können wiederum nur als Überraschung oder Ereignis auftreten (sonst wären sie nicht neu), als Ergebnis einer unpersönlichen Selbstorganisation und Mitwirkung der Dinge. Worauf Sam Ginn treffen muss, ist - mit Martin Heidegger formuliert - ein Moment der Selbstentbergung des Seins, ${ }^{24}$ in dem das (unbewusste) Machen, das Suchen und Tasten, der Reflexion vorausgeht. Und nicht nur für Heidegger ist die Technik in der modernen Kultur der Ort, wo es zu derartigen Wahrheitsereignissen kommen kann. In jüngerer Zeit hat vor allem der Wissenschaftshistoriker Hans Jörg Rheinberger gezeigt, wie sehr dieses unerwartete, nicht ableitbare Moment, das „präzedenzlose Ereignis“, ${ }^{25}$ nicht die Entdeckung, den Kern von Experimentalität ausmacht, die für neuzeitliche Wissenschaft von Anfang an konstitutiv gewesen ist.

23 Michel Serres, Vorwort, in: Authier/ders. (Hrsg.), Elemente einer Geschichte der Wissenschaft, 1994, 11, 35.

24 Vgl. dazu auch Hans Ulrich Gumbrecht, Nachwort. Mediengeschichte als Wahrheitsereignis. Zur Singularität von Friedrich A. Kittlers Werk, in: Kittler, Die Wahrheit der technischen Welt, 2013, 396.

25 Hans Jörg Rheinberger, Experimentalität, 15. 


\section{Folgen für das förmliche Recht: (Selbstlernende) Gesetzes-Fertigung}

Es ist davon auszugehen, dass die informationstechnologische Kultur tatsächlich mit einer Disruption, einer umstürzenden Neufundierung der Wissensbestände der Gesellschaft, einhergeht. Wie aber könnte eine auf Sam Ginn und die Welt des Silicon-Valley reagierende Form des Rechts aussehen, eine Rechtsform, von der eine „entwicklungsadäquate Gerechtigkeit" für die informationstechnologische Kultur ausgehen könnte?

Ein Teil der Antwort wird schnell deutlich: Wenn Staaten, Nationen oder Regionen an der Weiterentwicklung der Informationstechnologie partizipieren wollen, brauchen sie Typen wie Sam Ginn. Das heißt, sie brauchen vor allem Institutionen wie Stanford als Universität und Hochtechnologiecluster wie das Silicon Valley, in denen freie und produktive Geister forschen und ihre Persönlichkeit entwickeln können. Dazu benötigt man nicht nur den formalen Schutz der Freiheit dieser Institutionen durch die Gewährleistung wissenschaftlicher Freiheit, sondern auch eine Kultur der Offenheit, ein „gesellschaftliches Klima“, das für die Idee des technischen und gesellschaftlichen Fortschritts empfänglich bleibt und in dem der kollektive Glaube vorherrscht, dass sich beispielsweise durch die Weiterentwicklung künstlicher Intelligenz in Zukunft viele nützliche Dinge realisieren lassen werden, wie beispielsweise die Verbesserung von Medikamenten zur Bekämpfung von Epidemien oder tödlich verlaufenden Krankheiten. Damit ist für das Öffentliche Recht vor allem der Bereich der Freiheitsrechte, der subjektiven Rechte oder Grundrechte angesprochen. Dieser Themenkomplex soll hier übersprungen werden, stattdessen werde ich mich auf eine verwaltungsrechtswissenschaftliche Fragestellung beschränken, auf die Frage nach der Form des Gesetzes und die Beziehung von Gesetz und Verwaltung. Was können Institutionen wie Gesetzgebung und Verwaltung in einer informationstechnologischen Kultur leisten, ja was bedeutet es in einer informationstechnologischen Kultur überhaupt, Gesetze zu geben und durch die Verwaltung umzusetzen?

Für Institutionen wie Gesetzgebung und Verwaltung lehrt uns die jüngere informationstechnologische Entwicklung folgendes: Das Öffentliche Recht kann sich heute nicht mehr an einem Modell oder Idealtypus orientieren, den man - in Anlehnung an Max Weber - den Idealtypus der „Herrschaft kraft Wissen“ nennen könnte. ${ }^{26}$ Dieses Modell geht vor dem Hintergrund der langen europäischen Geschichte der Monarchie davon aus, dass in der staatlichen Verwaltung ein gegenüber dem gesellschaftli-

26 Max Weber, Wirtschaft und Gesellschaft (1922), 1980, 129. 
chen Wissen überlegenes Wissen mehr oder weniger zentral akkumuliert werden kann. Demgegenüber sind wir heute mit der Erfahrung konfrontiert, dass neues Wissen heute in der Gesellschaft in einer zerstreuten dezentralen Form erzeugt wird, wie beispielsweise in Hochtechnologieclustern wie dem des Silicon Valley. Dieses Wissen ist so dynamisch und komplex, dass selbst diejenigen, die es produzieren, es eher finden als planmäßig konstruieren. Das heißt, es gibt immer schon eine nicht hintergehbare Informationsasymmetrie zwischen Gesellschaft und Staat - zu Lasten des Staates. In der Gesellschaft ist mehr informationstechnologische Intelligenz aggregiert als im Staat (der im Gegenteil große Probleme hat, sich auf die neuen digitalen Gegebenheiten einzustellen), ${ }^{27}$ und an dieser Asymmetrie wird sich in absehbarer Zukunft nicht sehr viel ändern.

Darauf aufbauend, ließe sich folgende Hypothese wagen: Das Gesetz wird auch in Zukunft ein Resultat menschlichen Handelns sein und bleiben, aber es kann nicht länger die Form eines konstruktiven Entwurfs annehmen, eines Designs, einer Planung, eines Programms, einer Gesetzmäßigkeit im strengen Sinn. Weil der Gesetzgeber die Vielfalt der Handlungsmöglichkeiten in den von der informationstechnologischen Kultur betroffenen Handlungsfeldern nicht ex-ante voraussehen kann, kann er auch keine sinnvollen ex ante Handlungsanweisungen formulieren. Das Gesetz muss vielmehr in die Zeit eintauchen und temporalisiert werden. Es müsste die Form einer laufenden „Gesetzes-Fertigung" annehmen, zu einem selbstlernenden System werden. Es müsste anfänglich vorhandene normative Löcher, ja eine anfänglich vorhandene normative Leere akzeptieren lernen, die erst mit Hilfe der Zeit ausgefüllt und Schritt für Schritt in normative Bestimmtheit - fühlend und tastend - transformiert werden kann. Es ginge, in der Terminologie von Ino Augsberg, um eine neuartige Relationierung von Normalität und Normativität, die mit einer „Umstellung der Perspektive von Zeit auf Zeitlichkeit qua Zeitigung“ einhergehen müsste. ${ }^{28}$

Die normativen Löcher und die normative Leere müssten besonders auf der Ebene der Gesetzgebung akzeptiert werden. Das erscheint auch vertretbar, soweit die normativen Löcher durch die staatliche Verwaltung, hybride Staat/Gesellschafts-Verwaltungskörper oder durch unmittelbar in der Gesellschaft angesiedelte Körper, also etwa durch große informationstech-

27 Vgl. Martin Schallbruch, Schwacher Staat im Netz. Wie die Digitalisierung den Staat in Frage stellt, 2018, insb. 127ff., 219ff.

28 Ino Augsberg, Die Normalität der Normativität, JZ 9 (2020), 425, 430; vgl. auch Thomas Vesting, Einbau von Zeit. Rechtsnormativität im relationalen Vertrag, Kritische Justiz 4 (2019), 626. 
nologische Unternehmen wie Apple, Google oder Facebook, selbst gefüllt werden können. Das heißt, öffentliche Verwaltung und private Unternehmen müssten in die Prozesse der normativen Fertigung des Gesetzes und seine praktische Anwendung und Umsetzung einbezogen werden; die Rechtsbildungsprozesse müssten in Zukunft wieder viel stärker in die Gesellschaft zurückverlagert werden, dorthin, wo sie vor der explosionsartigen Zunahme des parlamentarischen Gesetzgebungsrechts im 20. Jahrhundert ohnehin angesiedelt waren. Für Edvard Coke, einen der bedeutendsten Anwälte des modernen englischen common law, hatten soziale Normen als Gesetze eine höhere Autorität als die des englischen Königs; ${ }^{29}$ und noch für Bernhard Windscheid war das Gewohnheitsrecht im letzten Drittel des 19. Jahrhunderts die höchste Rechtsquelle. ${ }^{30}$ Das Konzept der Gesetzes-Fertigung ist also keine Neuerfindung, sondern kann sich auf eine lange liberale Tradition stützen und wäre - rechtstheoretisch gesehen eine Art Variante dessen, was Rudolf Wiethölter die „Recht-Fertigung eines Gesellschafts-Rechts“31 genannt hat oder was Karl-Heinz Ladeur als „Prozeduralisierung zweiter Ordnung“ beschreibt. ${ }^{32}$

\section{Zwischenüberlegung: Regulierte Selbstregulierung als frühe Variante (selbstlernender) Gesetzes-Fertigung}

Als eine frühe Variante der Rechtsform der (selbstlernenden) Gesetzes-Fertigung ließe sich die Aufwertung von Organisation und Verfahren im Kon-

29 Vernon L. Smith, Constructivist and Ecological Rationality in Economics, Nobel Prize Lecture, December 8, Stockholm 2002, 502.

30 Bernhard Windscheid, Lehrbuch des Pandektenrechts Bd. 1, Düsseldorf 1862, 38. Für Windscheid war die „Rechtsvernunft“ die höchste Quelle allen Rechts, die sich zunächst im Gewohnheitsrecht artikulierte. Anders sah es Windscheid allerdings in späteren Auflagen, in denen er einen allgemeinen Bedeutungsverlust des Gewohnheitsrechts im Verhältnis zur Gesetzgebung konstatierte.

31 Rudolf Wiethölter, Recht-Fertigungen eines Gesellschafts-Rechts, in: Zumbansen/ Amstutz (Hrsg.), Recht in Recht-Fertigungen, 101; vgl. auch Dan Wielsch, Medienregulierung durch Persönlichkeits- und Datenschutzrechte, JZ 3 (2020), 105, 114 (der die nichtstaatliche Regelbildung akzentuiert und eine „Art Entdeckungsprozess von Institutionen der rechtsförmigen Teilhabe an der Regelbildung sozialer Praxis“ einfordert).

32 Karl-Heinz Ladeur, Recht, Wissen, Kultur, 34, 132, 178ff.; Gunnar Folke Schuppert, Wissen, Governance, Recht: Von der kognitiven Dimension des Rechts zur rechtlichen Dimension des Wissens, 2019, 86; vgl. auch Thomas Vesting, Instituierte und konstituierte Normativität. Prozeduralisierung und multi-normative Systeme, in: Sheplyakova (Hrsg.), Prozeduralisierung des Rechts, 2018, 100. 
text von Modellen wie regulierte Selbstregulierung, Governance oder auch des Gewährleistungsstaates interpretieren. ${ }^{33}$ Auch in diesen Modellen geht es um eine Flexibilisierung der klassischen Form des allgemeinen Gesetzes zugunsten der normativen Fertigung des Gesetzes mit Hilfe von Zeit. Heute wird von regulierter Selbstregulierung meist in Kontexten gesprochen, in denen das Handeln privater Akteure zur Sicherung von öffentlichen Interessen und Belangen beitragen soll. Dabei wird meistens auf Managementkonzepte (Compliance) privater Wirtschaftsunternehmen oder an die Ersetzung staatlicher Überwachungstätigkeit im Rahmen des (europäisierten) Produktsicherheitsrechts verwiesen. ${ }^{34}$ Man könnte das Konzept der regulierten Selbstregulierung aber auch weiter fassen und bereits solche Fälle einbeziehen, in denen der Staat auf einer eher unbestimmten gesetzlichen Grundlage Aufgaben an öffentlich-rechtliche, gegenüber dem Staat verselbständigte Verwaltungskörper delegiert, die ihrerseits ein spezifisch gesellschaftliches Wissen aufnehmen, bündeln und zugleich die dahinter stehenden Interessen kompatibilisieren sollen. Das war beispielsweise ursprünglich die Aufgabe der binnenpluralistischen Gremien des öffentlichrechtlichen Rundfunks: Diese Gremien sollten für die Selbstorganisation des journalistischen Professionalismus in den Anstalten einen offenen Orientierungs- und Entwicklungsrahmen schaffen. ${ }^{35}$

Dieses Modell war in der alten Bundesrepublik viele Jahrzehnte erfolgreich. Gerade am Beispiel des Rundfunkrechts lässt sich jedoch die Grenze des Modells der regulierten Selbstregulierung und der ihr nahestehenden Konzepte aufzeigen: Es wird ein Ziel festgelegt, die Schaffung einer öffentlich-rechtlichen Rundfunkanstalt zur Produktion von ausgewogenen und sachlichen Programminhalten, die die in der Gesellschaft zerstreuten Prozesse öffentlicher Meinungsbildung auf die Reproduktionsfähigkeit der parlamentarischen Demokratie ausrichten sollen. Diese normative Festlegung wurde schon durch das Aufkommen des privaten Rundfunks auf eine harte Probe gestellt, aber es wird noch viel schwieriger werden, mit einer solchen Strategie die überraschende Wendung zu bewältigen, die die informationstechnologische Kultur durch den Aufstieg von Plattformen

33 Vgl. allgemein Wolfgang Hoffmann-Riem, Innovation und Recht - Recht und Innovation. Recht im Ensemble seiner Kontexte, 2016, 374ff.; Gunnar Folke Schuppert, Governance und Rechtsetzung. Grundfragen einer modernen Regelungsgesellschaft, 2011, 253ff. (zur Regulierung); ders., Wissen, Governance, Recht.

34 Hofmann-Riem, Innovation und Recht - Recht und Innovation, 375; Schuppert, Wissen, Governance, Recht, 65, 67; Martin Eifert, Regulierte Selbstregulierung, Die Verwaltung, Beiheft 4, 2001, 137.

35 Vgl. Martin Stock, Medienfreiheit als Funktionsgrundrecht, 1985. 
wie YouTube, Netflix, Amazon Prime oder von Content-Kuratoren wie Google genommen hat. Mit dieser plötzlichen Wendung hat vor allem das herkömmliche Programmfernsehen seine Dominanz als öffentlicher Distributionskanal für politische (und sonstige) Informationen verloren. Jeder kann jetzt mit Hilfe eines Handys eine große Öffentlichkeit durch einen Tweet oder eine Chat Group erzeugen: Donald Trump hat 71 Millionen Follower auf Twitter, Cristiano Ronaldo 200 Millionen Follower auf Instagram, usw. Der Staat aber hat sich im Kern (von der Regulierung des privaten Rundfunks einmal abgesehen) auf die Förderung und Sicherung von öffentlich-rechtlichen Rundfunkanstalten festgelegt, während die technologische Kreativität und Intelligenz der Gesellschaft andere Wege gegangen ist.

Nimmt man diese Entwicklung als Beispiel, könnte man verallgemeinernd schlussfolgern, dass das (hier zugegebenermaßen sehr grob skizzierte) Modell der regulierten Selbstregulierung seine Grenze darin findet, dass die gesetzlichen Ziele und Mittel zu einem bestimmten Zeitpunkt zu starr fixiert sind. ${ }^{36}$ Statt in einem experimentellen Setting auf das Ereignishafte und Unvorwegnehmbare, die Offenheit der Technologieentwicklung, vorbereitet zu sein, wird das Modell der regulierten Selbstregulierung vielleicht noch zu sehr von der Idee der „Herrschaft kraft Wissen“ okkupiert, von der Vorstellung einer planmäßig langfristigen, rahmenförmig festgelegten und gesicherten Verlaufsgeschichte eines zu verwirklichenden Projekts, wie eben der Versorgung der Bevölkerung mit demokratiefreundlichen Informationsinhalten durch öffentlich-rechtliche Rundfunkanstalten. Wenn sich die „Wahrheit“ der öffentlichen Kommunikation aufgrund neuer und bis vor kurzem unvorhersehbarer technologischer Entwicklungen aber woanders als im herkömmlichen Programmfernsehen „entbirgt“, dann hat dieses Modell ein faktisches Problem, das auf Dauer nicht allein durch das Medienrecht bewältigt oder kompensiert werden kann. Auch die herkömmliche Rundfunk- und Medienrechtsprechung des Bundesverfassungsgerichts stößt dann unweigerlich an Grenzen oder läuft Gefahr selbst zu starr zu werden, die technologische Entwicklung zu bremsen und den Staat immer weiter von der Technikentwicklung der Gesellschaft abzukoppeln.

36 Vgl. aber jüngst Gunnar Folke Schuppert, Wissen, Governance, Recht (der die Governance-Perspektive für die Wissensproblematik öffnet); in diese Richtung auch Wolfgang Hoffmann-Riem, Innovation und Recht - Recht und Innovation, $303 \mathrm{ff}$. 


\section{Das Beispiel der Gesundheitsregulierung}

Wie die problematische Seite des tradierten Modells „Herrschaft kraft Wissen" aussieht, lässt sich auch am Beispiel des Gesundheitsrechts aufzeigen. Im Gesundheitsrecht, der medizinisch-gesundheitlichen Versorgung der Bevölkerung durch niedergelassene Ärzte oder durch Krankenhausbehandlung, geht es regelmäßig um (Rechts-)Konflikte, die medizinische Fragen betreffen, in denen es bei allen therapeutischen Maßnahmen, Mitteln und Methoden im Kern um die Frage geht, ob „bestimmte Methoden oder Mittel wirksam und bestimmte Vorgehensweisen riskant oder unbedenklich sind “. ${ }^{37} \mathrm{Ob}$ das der Fall ist oder nicht, wird im Gesundheitsrecht grundsätzlich dadurch entschieden, dass auf übereinstimmende Richtigkeitsüberzeugungen referiert wird, auf den Stand der medizinischen Erkenntnisse, der in den maßgeblichen ärztlichen Kreisen allgemein anerkannt ist. ${ }^{38}$ Es wird also wie im liberalen Polizeirecht ein gesicherter Wissensbestand unterstellt, ein ärztliches common knowledge, ${ }^{39}$ anhand dessen bestimmte Behandlungsmaßnahmen als standardgemäß eingestuft werden und andere nicht. ${ }^{40}$

Die Medizin selbst wird dieser Vorstellung von einem allgemein anerkannten gesicherten Wissensbestand aber schon lange nicht mehr gerecht. Seit einigen Jahrzehnten vollzieht sich im Gesundheitssektor eine Selbsttransformation der Wissenserzeugung, die von der erfahrungsgeleiteten zur datenbasierten Medizin führt. Dadurch wird das erfahrungsgeleitete Wissen des einzelnen Arztes, das sich nur langsam - durch die akademische Ausbildung und aufgrund praktischer Erfahrungen - ändert, nicht abgeschafft oder überflüssig, aber die medizinische Wissensordnung als sol-

37 Friedhelm Hase, Die Komplexität und Veränderlichkeit medizinischen Wissens als Herausforderung der juristischen Hermeneutik: Gesundheitsrecht zwischen gesetzlicher Normierung und 'autonomer' Selbststeuerung des Versorgungssystems, in: Broemel/ Pilniok (Hrsg.), Die digitale Gesellschaft als Herausforderung des Rechts, i.E., Manuskript 141ff., 146.

38 Hase, ebd.

39 Vgl. Karl-Heinz Ladeur, Wissensgenerierung in und durch Recht - und das Problem der "evidenzbasierten Medizin", GesR 2011, 455; Friedhelm Hase, Sozialrecht und die Integration gesellschaftlichen Wissens, in: Masuch u.a. (Hrsg.), Grundlagen und Herausforderungen des Sozialstaats - Denkschrift 60 Jahre Bundessozialgericht, Bd. 1 2014, 423.

40 Vgl. Benedikt Buchner, Wissen und Standard - Herausforderungen für Wissensgenerierung und -verarbeitung bei der medizinischen Standardfestlegung, in: ders./ Ladeur (Hrsg.), Wissensgenerierung und -verarbeitung im Gesundheits- und Sozialrecht, 2016, 63. 
che wird komplexer: Es existieren jetzt verschiedene Wissensarten nebeneinander. Es entsteht zunächst ein Nebeneinander von einzelärztlichem Erfahrungswissen und dem organisierten und spezialisierten Wissen der evidenzbasierten Medizin. ${ }^{41}$ Deren Testverfahren - randomized clinical trials haben sich bereits einen gehörigen Schritt von der Vorstellung eines gesicherten ärztlichen Wissens entfernt und ersetzen dieses durch die Aussagekraft statistischer Konzepte. Der nächste Evolutionsschritt wird durch die datenbasierte Medizin bestimmt, die auf dem Gebrauch von hochavancierten informationstechnologischen Architekturen beruht und damit über die evidenzbasierte Medizin und ihre Spezialisierungs- und Standardisierungsleistungen hinausführt. Die Dynamik wird sich künftig noch dadurch weiter beschleunigen, dass eine Fusion von Biotechnologie und Informationstechnologie denkbar geworden ist, die bislang ungeahnte Möglichkeiten der Heilung von Krankheiten eröffnen könnte.

Damit wird folgende kognitive Struktur im Gesundheitssystem erkennbar: Das Wissen des einzelnen Arztes beruht auf einer mit anderen geteilten gemeinsamen Erfahrung, einer Selbstorganisation des gesellschaftlichen Wissens, in dessen Referenzrahmen sich auch die individuelle ärztliche Intuition bewegt. Die evidenzbasierte Medizin basiert demgegenüber auf einem in Krankenhäusern und Forschungseinrichtungen spezialisierten Gruppenwissen. Die datenbasierte informationstechnologische Medizin erneuert die Formen der Generierung und die Muster des medizinischen Wissens nun ein weiteres Mal: Die Informations- und Wissenserzeugung spielt sich jetzt in offenen Netzwerken ab, die auch die Grenzen des medizinischen Wissens selbst durchlässig werden lassen. Das Wissen wird gewissermaßen laufend dekontextualisiert und mittels leistungsfähiger Computer und formaler Algorithmen immer wieder neu zusammengestellt. ${ }^{42}$ Damit wird vermutlich eine Zunahme von unvorhersehbaren Entwicklungen im Medizinsektor einhergehen, ${ }^{43}$ wenn man in Rechnung stellt, dass die besondere Bedeutung der neuen algorithmenbasierten Wis-

41 Vgl. Dominik Roters, Wissensgenerierung und -verwertung nach $₫ 2$ Abs. 1 S. 3 SGB V, in: Buchner/Ladeur (Hrsg.), Wissensgenerierung und -verarbeitung im Gesundheits- und Sozialrecht, 31; Steffen Augsberg, Kooperative Wissensgenerierung im Gesundheitsrecht, GesR 2012, 595; vgl. auch Ino Augsberg, Evidence-based Law im Sozial- und Gesundheitsrecht?, in: Buchner/Ladeur (Hrsg.), Wissensgenerierung und -verarbeitung im Gesundheits- und Sozialrecht, 73.

42 Vgl. Karl-Heinz Ladeur, Der Wandel des technischen und medizinischen Wissens und die Notwendigkeit der Herausbildung einer entscheidungsorientierten „Heuristik“, Manuskript 2017, i.E.

43 Wolfgang Hoffmann-Riem, Innovation und Recht - Recht und Innovation, 616. 
senstechnologien gerade in der Fruchtbarkeit der ungewissen Verwendung großer Datenmengen liegt, d.h. die Daten können immer wieder zu in der Gegenwart noch unbekannten Zwecken genutzt werden. ${ }^{44}$

Je mehr sich das medizinische Wissen jedoch von der praktischen Alltagserfahrung der Ärzte selbst ablöst und seine interne Komplexität durch elektronisch generiertes Wissen steigert, umso mehr wird dieses Wissen auch für den Gesetzgeber undurchschaubarer und unzugänglicher. Weil die Bedeutung gesellschaftlicher (privater) Wissensgenerierung und ihre Selbstorganisation in der Hightech-Medizin dramatisch wächst, gelangt die tradierte Form der „Herrschaft kraft Wissen“ im Gesundheitssektor an strukturelle Grenzen. Das trifft vor allem die Vorstellung einer parlamentarischen ex-ante Gesetzgebung, mit der Folge, dass die Gesundheitsverwaltung nicht mehr an einen zentral gebildeten gesetzgeberischen Willen gebunden werden kann.

Es spricht deshalb alles dafür, dass die gesellschaftliche Wissensgenerierung und die sie begleitende selbstregulative Ordnungs- und Regelbildung in einer neuen Weise in die herkömmliche rechtliche Regulierung und Entscheidungsfindung integriert werden muss: Die staatliche Rechtssetzung mag im Gesundheitssektor auch künftig eine Art Rahmen vorgeben können, aber dieser Rahmen kann allenfalls ein Rahmen auf Rädern sein: Der Staat ist heute wie nie zuvor auf eine fortlaufende Abstützung durch gesellschaftliche Selbstorganisationsmechanismen angewiesen, wie sie im Gesundheitsrecht nicht zuletzt durch Selbstverwaltungseinrichtungen, vor allem durch den Gemeinsamen Bundesausschuss (der Organisation der Ärzte, Krankenhäuser und gesetzlichen Krankenversicherungen - G-BA, $\$ 91$ SGB V), bekannt sind. Neu ist aber, dass die gesetzliche und untergesetzliche Regelbildung in Zukunft zunehmend dialogisch-interaktiv aufeinander bezogen werden müssen: Das Gesetz lernt „von unten“, im Verlauf eines Prozesses, in dem Erfahrungen gesammelt und normative Löcher Schritt für Schritt ausgefüllt werden. ${ }^{45}$

44 Vgl. Roland Broemel/Hans-Heinrich Trute, Alles nur Datenschutz? Zur rechtlichen Regulierung algorithmenbasierter Wissensgenerierung, Berliner Debatte Initial 27 (2016), 50, 52.

45 Friedhelm Hase, Die Komplexität und Veränderlichkeit medizinischen Wissens als Herausforderung der juristischen Hermeneutik, in: Broemel/Pilniok (Hrsg.), Die digitale Gesellschaft als Herausforderungen für das Recht in der Demokratie, 2020; ders., Die Komplexität des Wissens und die Legitimation rechtlicher Normsetzung, in: Buchner/Ladeur (Hrsg.), Wissensgenerierung und -verarbeitung im Gesundheits- und Sozialrecht, 125, 134f. 
Mit anderen Worten: Mit dem Aufstieg der datenbasierten Medizin verändert sich das Verhältnis des im Gesundheitssektor erzeugten medizinischen Wissens zum parlamentarischen Gesetz und zu den sonstigen Normbeständen. Die Komplexität des Gesundheitssektors sprengt vor allem die Vorstellung der Zentralität eines Gesetzgebers und der Beherrschung dieser Komplexität durch das allgemeine Gesetz. Ja, es wird geradezu unwahrscheinlich, dass die Herausforderung der verflüssigten Wirklichkeit der datenbasierten Medizin durch allgemeine, ex ante formulierte Gesetze überhaupt bewältigt werden könnte. Die Vorstellung, dass die Wissenserzeugung im Gesundheitssektor im Allgemeinen und die sachverständige Prüfung des diagnostischen und therapeutischen Nutzens neuer Untersuchungs- und Behandlungsmethoden im Besonderen einer gesetzlichen Ausgestaltung bedürfe und beaufsichtigt werden müsse, wie es das Bundesverfassungsgericht im Kontext der nach $₫ 135$ SGB V vorgesehen Verfahren des G-BA offensichtlich unterstellt - wenn auch nicht näher geprüft hat, ${ }^{46}$ geht an dieser Entwicklung vorbei. Das Bundesverfassungsgericht mahnt eine Form der demokratischen Legitimation der Wissensgenerierung im Gesundheitssektor an, die angesichts der erreichten Komplexität des Wissens nicht mehr möglich ist. Aus dem staatlichen Gesetz muss vielmehr eine (selbstlernende) iterative Gesetzes-Fertigung werden. ${ }^{47}$

\section{Das Beispiel der Bankenregulierung}

Die Notwendigkeit der transformierenden Verarbeitung eines hochdynamischen gesellschaftlichen Wissens beherrscht ferner das Bankenaufsichtsrecht und insbesondere das Feld der Finanzmarktaufsicht. ${ }^{48}$ Die weltweite Liberalisierung der Finanzmärkte seit den 1970er Jahren und die Aufwer-

46 Vgl. BVerfG, Beschluss des Ersten Senats vom 6. Dezember 2005 - 1 BvR 347/98 -, juris Rn. 60; ferner etwa BVerfG, Beschluss der 1. Kammer des Ersten Senats vom 2. Mai 2018 - 1 BvR 3042/14 -, juris, Rn. 25 ff.

47 Vgl. auch Karl-Heinz Ladeur, Die Textualität des Rechts. Zur poststrukturalistischen Kritik des Rechts, 2016, 309.

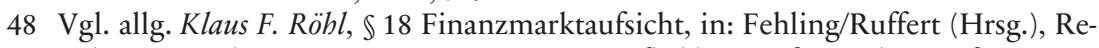
gulierungsrecht, 2010, 1003; Ann-Katrin Kaufhold, Transfer und Transformation ökonomischen Wissens im Recht der Bankenaufsicht, in: Augsberg (Hrsg.), Extrajuridisches Wissen, 2013, 151, 156ff.; Katharina Reiling, Der Hybride. Administrative Wissensorganisation im privaten Bereich, 2016, 137ff. (zur Bankenaufsicht durch den Bankenprüfer); Gunnar Folke Schuppert, Wissen, Governance, Recht, $59 \mathrm{ff}$. 
tung der Kapitalmärkte für die Unternehmensfinanzierung ${ }^{49}$ sind mit einer enormen Beschleunigung der Produktion hochspeziellen ökonomischen Wissens einhergegangen. Insbesondere die mathematische und computergestützte Modellierung von risikoreichen Anlagestrategien ist ein relativ junges Phänomen, deren kaskadenartige Verbreitung ein Auslöser für die Finanzmarktkrise von 2008 gewesen sein dürfte. ${ }^{50}$

Finanzprodukte werden in den epistemischen Gemeinschaften ${ }^{51}$ des Investmentbanking erzeugt. In der Finanzmarktkrise haben sich aber selbst hochriskante Anleihekonstruktionen rasch verbreiten und Nachahmer finden können, weil eine Kontrolle durch die wechselseitige Beobachtung von Banken nicht stattgefunden hat; die Modellierungen im Investmentbanking wurden offensichtlich schon von der Leitungsebene der Banken und dessen Top-Management nicht verstanden. ${ }^{52}$ Das Bonus-System dürfte die Ansteckungs- und Nachahmungseffekte dieser komplexen und opaken Finanzprodukte dann noch einmal verstärkt haben. Wie immer es aber auch im Einzelnen gewesen sein mag, das Beispiel der Finanzmarktkrise belegt jedenfalls eindrucksvoll, dass das Recht der Finanzmarktaufsicht seit geraumer Zeit mit der rasanten Selbstveränderung einer gesellschaftlichen Wissensordnung konfrontiert ist, die für die staatliche Verwaltung nicht unmittelbar zugänglich ist. Daraus resultiert die Schwierigkeit, die Eigenrationalität des Verwaltungsrechts gegenüber dem in Banken und Finanzinstituten vorhandenen außerrechtlichen Wissen in Anschlag bringen zu können. ${ }^{53}$

Für die Banken- und Finanzmarktaufsicht sind in Deutschland die Bundesbank und die Bundesanstalt für Finanzdienstleistungsaufsicht (BaFin) zuständig. Seit dem Ende der 1980er Jahre wird insbesondere die Finanzmarktaufsicht durch die Vorgaben der Baseler Abkommen bestimmt.

49 Für Deutschland vgl. Tobias Tröger, Vom Rheinischen Kapitalismus zum Kapitalmarktrecht (und wieder zurück?), in: Duve (Hrsg.), Rechtswissenschaft in der Berliner Republik, 2018, 664ff.

50 Karl-Heinz Ladeur, Recht - Wissen - Kultur, 96; und die Beiträge in: Kjaer/Teubner/Febbrajo (Hrsg.), Financial Crisis in Constitutional Perspective: The Dark Side of Functional Differentiation, Oxford 2011.

51 Dazu etwa Katharina Reiling, Der Hybride, $330 \mathrm{ff}$.

52 Larry R. Wray, The Rise and Fall of Money Manager Capitalism: A Minskian Approach, Cambridge Journal of Economics 33 (2009), 807, 820.

53 Vgl. Ann-Katrin Kaufhold, Transfer und Transformation ökonomischen Wissens im Recht der Bankenaufsicht, 153; vgl. auch allg. Ivo Appel, Bedeutung außerrechtlicher Wissensbestände für das Management von Unsicherheit und Nichtwissen, in: Hill/Schliesky (Hrsg.), Management von Unsicherheit und Nichtwissen, 2016, 113. 
Während noch in den 1970er Jahren eine Regulierung etwa von Marktstrukturen, Anlagen und Einlagenzinsen üblich war, hat der Baseler Ausschuss für Bankenaufsicht (Basel Committee on Banking Supervision) die Eigenkapitalanforderungen der Banken zum zentralen Instrument der Bankenregulierung gemacht. ${ }^{54}$ Seit Basel II ist damit eine Öffnung des Verwaltungsverfahrens für ökonomisches Wissen einhergegangen, weil die aufsichtsunterworfenen Banken ihre selbstentwickelten Risikobewertungsverfahren in diese Prüfung einführen können bzw. die bankeninternen Bewertungen von der Aufsicht berücksichtigt werden müssen; die Banken können die Bonität ihrer Schuldner mit Hilfe ihrer eigenen betriebswirtschaftlichen Risikomodelle bewerten (Internal Ratings Based Approach). ${ }^{55}$

Hier liegt das schwer zu bewältigende Problem. Da das übergreifende Ziel der Bankenaufsicht in der Gewährleistung der Funktionsfähigkeit des Bankensystems liegt, kann die staatliche Bankenaufsicht ohne das im Bankensystem generierte Wissen gar keine Einschätzungen zur Funktionssicherung liefern. Die staatliche Aufsicht muss das gesellschaftliche Wissen vielmehr rezipieren und über ein normatives Konkretisierungsverfahren in eigene Bewertungen transformieren. ${ }^{56}$ Dieses Verfahren ist aber alles andere als einfach auszugestalten und beschränkt die Selektion des rezipierten Wissens momentan allein auf den Sachverstand international tätiger Großbanken. Auch die normative Transformation, insbesondere die Gewichtung der Informationen, die die Risikoeinschätzung betreffen, ist verfahrensrechtlich sehr einfach strukturiert. ${ }^{57} \mathrm{~W}$ ie immer mögliche Lösungsvorschläge und Verbesserungen auch aussehen mögen, der Lösungsvorschlag kann nicht das Resultat eines konstruktiven Entwurfs, eines Designs, einer Planung oder eines Programms sein, sondern muss in einem Modell verankert werden, dass das Prozessieren der Verwaltung in und mit Wissensbeständen, die sie selbst nicht erzeugen kann, berücksichtigt.

54 Ann-Katrin Kaufhold, Transfer und Transformation ökonomischen Wissens im Recht der Bankenaufsicht, 153f., 158, 167ff.; zum Hintergrund vgl. auch Katharina Pistor, The Code of Capital. How the Law Creates Wealth and Inequality, Princeton (N.J.) 2019, 143.

55 Kaufhold, Transfer und Transformation ökonomischen Wissens im Recht der Bankenaufsicht, 154, 169ff.; Klaus F. Röhl, $\mathbb{1 8}$ Finanzmarktaufsicht, Rn. 67.

56 Kaufhold, Transfer und Transformation ökonomischen Wissens im Recht der Bankenaufsicht, 159f.

57 Kaufhold, ebd., $169 \mathrm{ff}$. 


\section{Wandel der rechtswissenschaftlichen Methode}

Die Methodendiskussion im Verwaltungsrecht ist in der jüngeren Zeit stark durch die Entgegensetzung von textgebundener Interpretationswissenschaft und rechtssetzungsorientierter Handlungs- und Entscheidungswissenschaft bestimmt worden. Diese Differenzierung soll hier aber nicht noch einmal kommentiert werden. ${ }^{58}$ Stattdessen möchte ich abschließend lediglich die These formulieren, dass die entscheidende Herausforderung für die Methode der Verwaltungsrechtswissenschaft in dem liegt, was man den Umgang mit rechtlichen Institutionen nennen könnte: Das westliche liberale Recht basiert seit jeher auf stillschweigenden kulturellen und gesellschaftlichen Voraussetzungen, beispielsweise auf Gewohnheiten, sozialen Normen und einer Mitwirkung der Dinge. Diese Voraussetzungen müssen künftig wieder stärker in das Blickfeld der Rechtswissenschaft geraten. Die Juristen müssen wieder lernen, dass rechtliche Institutionen nicht-rechtliche Voraussetzungen haben, deren Wandel Institutionen auf die Probe stellen und auch ganz obsolet machen kann. Dies ist hier im Hinblick auf die Wissensgenerierung für einige verwaltungsrechtliche Handlungsfelder anschaulich gemacht worden. Mit der informationstechnologischen Kultur geht ein tiefgreifender gesellschaftlicher Umbruch einher, der auch die Verwaltungsrechtswissenschaft dazu nötigt, selbst Beiträge zur Konstruktion neuartiger Institutionen der Gesetzes-Fertigung zu leisten. Es wäre jedenfalls fatal zu glauben, dass diese neuen Institutionen ohne wissenschaftliche Anstrengungen, Vorüberlegungen und Vorschläge primär oder allein durch den Gesetzgeber oder gar die Gerichte geschaffen werden könnten. 
